\title{
The Effect of Autophagic Activity on the Function of Apheresis Platelets and on the Efficacy of Clinical Platelet Transfusion
}

\author{
Hao Tang ${ }^{a}$ Meng Gao ${ }^{a}$ Yunfeng $\mathrm{Fu}^{a}$ Rong Gui ${ }^{a}$ Xianjun Ma ${ }^{\mathrm{b}}$ \\ ${ }^{a}$ Department of Blood Transfusion, The Third Xiangya Hospital, Central South University, Changsha, China; \\ ${ }^{\mathrm{b}}$ Department of Blood Transfusion, Qilu Hospital of Shandong University, Jinan, China
}

\section{Keywords}

Apheresis platelets - Autophagy $\cdot$ Storage duration $\cdot$ ABO blood group - Efficacy of clinical platelet transfusion

\begin{abstract}
Platelet activation and survival jointly determine the efficacy of clinical platelet transfusion. This study aimed to discuss the effect of autophagic activity on activation and aggregation of apheresis platelets and on the efficacy of clinical platelet transfusion. In this study, we investigated the effects of autophagic activity of apheresis platelets for different blood types and after different storage durations on platelet activation and aggregation functions. By Western blot, immunofluorescence, and RT-qPCR detection, we found that with the prolongation of the storage duration, the expressions of both autophagy-related proteins and genes were upregulated in apheresis platelets and their expressions were insignificantly higher in the apheresis platelets of type $A$ and $O$ blood than in those of type $B$ and type $A B$ blood. After RAPA/IGF-1 pretreatment, there was a significant increase/reduction in autophagic activity. After RAPA and IGF1 pretreatment, an opposite variation trend was observed with platelet activation and aggregation. Autophagic activity of platelets correlated negatively with the efficacy of clinical platelet transfusion. These research findings provide a theoretical basis for effective clinical platelet transfusion.
\end{abstract}

(c) 2020 S. Karger AG, Basel

Hao Tang and Meng Gao contributed equally to this work. 
platelet transfusion or even leading to transfusion refractoriness [4]. Therefore, platelet activation is an important influence factor for the efficacy of platelet transfusion. Nevertheless, the specific mechanism of platelet activation has not been fully clarified and needs to be further explored.

Autophagy is the most important means of digestion and degradation in eukaryotes, which decomposes the ageing, damaged, and degenerative organelles and longlived proteins to maintain homeostasis [5]. Autophagy is closely associated with many physiopathological processes, such as host defense, cell survival and ageing, autoimmune diseases, neurodegenerative diseases, and cancer [6-8]. Earlier studies on autophagy mainly concentrated on karyotes, and rarely on akaryotes. Over the past 20 years, it has been recognized that autophagy is closely related to erythropoiesis and hematological diseases $[9,10]$. In the 1970 s, electron microscopy found that platelets in benign tumors had structures similar to autophagosomes, which is evidence of the autophagic activity of platelets [11]. Subsequent studies have shown that platelet autophagy can be induced by hunger or rapamycin and that autophagy defect may cause platelet aggregation and adhesion [12]. The existing studies are generally concerned with fresh platelets. The autophagic level in apheresis platelets and the effect of autophagic activity on platelet activation and aggregation and clinical platelet transfusion are not fully known in vitro. This study investigated the effects of autophagic activity of apheresis platelets for different blood types and after different storage durations on platelet activation and aggregation functions. The research findings provide a theoretical basis for effective clinical platelet transfusion.

\section{Materials and Methods}

\section{Culture and Passage of Bone Mesenchymal Stem Cells}

Bone mesenchymal stem cells (BMSCs) were provided by our laboratory and inoculated to Dulbecco's modified eagle medium (Hyclone, USA) containing 10\% fetal bovine serum (ExCell Biology, China). The surface of the culture medium was disinfected with alcohol, and the cells were cultured in an $\mathrm{CO}_{2}$ incubator $\left(37^{\circ} \mathrm{C}, 5 \% \mathrm{CO}_{2}\right)$. Once the BMSCs had grown to $80 \%$ confluence, the culture fluid was discarded. The cells were washed with $1 \times$ phosphate buffer saline (PBS; Wellbio, China) 3 times and digested with $0.25 \%$ trypsin (Invitrogen, USA) for $1 \mathrm{~min}$. Cell passage was performed immediately after digestion. The digested BMSCs were divided into two portions, which were transferred to two new culture discs. Fresh culture medium was added, and the culture medium was replaced regularly. The growth morphology of BMSCs was observed under an inverted microscope.

Detection of Proteins Related to the MTOR and JAK/STAT Signaling Pathway

The expressions of proteins related to the mammalian target of rapamycin (mTOR) and janus kinase (JAK)/signal transducer and activator of transcription(STAT) signaling pathway, including $\mathrm{p}$ mTOR, p-AKT, p-PI3K, p-JAK1, and p-STAT1, were detected before and after pretreatment with rapamycin (RAPA, $200 \mathrm{nM} / \mathrm{L}$; Sigma-Aldrich, USA) and insulin-like growth factor 1 (IGF-1, 50 nM/L; Sigma-Aldrich, USA) by Western blot, respectively. Total proteins were extracted from the cells of each group and quantified using bicinchoninic acid assay (Abcam, UK). The proteins were separated by sodium dodecylsulphate polyacrylamide gel electrophoresis (SDS-PAGE) and then transferred to a polyvinylidene fluoride membrane, which was sealed with tris-HCl-tween (TBST; Sigma, USA) containing 5\% defatted milk for $1 \mathrm{~h}$. Anti-p-mTOR, anti-p-AKT, anti-p-PI3K, anti-p-JAK1, and anti-p-STAT1 (all purchased from Abcam, UK) were respectively added to incubate the cells at $4{ }^{\circ} \mathrm{C}$ overnight. After washing the cells with TBST for 3 times, HRP-labelled secondary antibodies (Proteintech, USA) were added for co-culture for 45-60 min. Next, ECL reagent (Thermo Pierce, USA) was added to incubate the membrane for 3 min. The gels were exposed to X-ray with development and washing. The protein contents were analyzed.

\section{Detection of Autophagic Activities}

Immunofluorescence detection was performed to determine the expressions of Beclin 1 and LC3 in the stored platelets. Cell slides were prepared and fixed with $4 \%$ paraformaldehyde (SigmaAldrich, USA) for $30 \mathrm{~min}$. After transparentization with $0.5 \%$ triton X-100 (Sigma-Aldrich, USA) for $30 \mathrm{~min}$, the slides were sealed with $10 \%$ normal goat serum (ExCell Biology, China) for $1 \mathrm{~h}$. Then LC3 antibody (1:50 dilution; Proteintech, USA) and Beclin 1 antibody (Proteintech, USA) was added to incubate the cells at $4{ }^{\circ} \mathrm{C}$ overnight. After washing with PBS 3 times, 100-200 $\mu \mathrm{L}$ of antirabbit IgG-labelled fluorescent antibody (Proteintech, USA) was added, and the cells were incubated at $37^{\circ} \mathrm{C}$ for $30 \mathrm{~min}$. This was followed by washing with PBS 3 times. The slides were sealed with $90 \%$ glycerol and observed under a fluorescence microscope.

Western blot was used to detect the expressions of Beclin 1 and LC3-II in each group. Total proteins were extracted from cells in each group and quantified by bicinchoninic acid assay. Proteins were separated by SDS-PAGE and then transferred to a polyvinylidene fluoride membrane, which was sealed with TBST containing 5\% defatted milk for $1 \mathrm{~h}$. LC3 and Beclin 1 antibody (Proteintech, USA) were added to incubate the cells at $4{ }^{\circ} \mathrm{C}$ overnight. After washing with PBS 3 times, the HRP-labelled secondary antibodies (Proteintech, USA) were added for co-culture for 45-60 min, followed by addition of ECL reagent to incubate the cells for $3 \mathrm{~min}$. The gels were exposed to X-ray with development and washing. The protein contents were analyzed.

Expressions of Beclin 1 and LC3 genes in each group were detected by RT-qPCR. Total RNA extraction was performed using Trizol reagent (Invitrogen, USA). RNA was reversely transcribed into cDNA using total mRNA as the template. The primers (Table 1) were designed using Primer5 software for amplification of the target genes and synthesized by Shanghai Shenggong Bioengineering Co., Ltd. Gel electrophoresis was performed to visualize the results of PCR.

\section{Detection of Activation of Apheresis Platelets by Flow} Cytometry

The apheresis platelet suspensions were centrifuged at 4,000 revolutions per minute (rpm) for $15 \mathrm{~min}$, and the supernatant was discarded. The platelet precipitate was washed with PBS 3 times, 5 min each time. Then, it was pretreated with RAPA $(200 \mathrm{~nm} / \mathrm{L})$ and IGF-1 (50 nM/L), respectively. The pretreated platelets were collected and adjusted to a density of $1 \times 10^{6} / \mathrm{mL}$. After washing with PBS twice, centrifugation was performed at $1,000 \mathrm{rpm}$ for $5 \mathrm{~min}$. The supernatant was discarded, and the cells were resus- 
Table 1. Primers designed for $q R T-P C R$ validation of candidate proteins

\begin{tabular}{ll}
\hline \multicolumn{2}{c}{ Primer } \\
\hline LC3 & \\
Forward & 5'-GCGTCTCCACACCAATCTCA-3' \\
Reverse & $5^{\prime}$-GACAATTTCATCCCGAACGTCT-3' \\
Beclin 1 & \\
Forward & $5^{\prime}$-CATGGAGAACCTCAGCCGAA-3' \\
Reverse & $5^{\prime}$-ACAGCGTTTGTAGTTCTGACAC-3' \\
$\begin{array}{c}\text {-actin } \\
\text { Forward } \\
\text { Reverse }\end{array}$ & $5^{\prime}$-ACCCTGAAGTACCCCATCGAG-3' \\
\hline
\end{tabular}

pended in buffer. Then, $100 \mu \mathrm{L}$ platelet suspension was collected, $5 \mu \mathrm{L} \mathrm{PE}$ and APC dyes were added, respectively, and mixed well. The reaction proceeded at room temperature in the dark for 10 min. Subsequently, anti-CD41a (BD, USA) and anti-CD62p (BD, USA) were added. The reaction proceeded at room temperature in the dark for $30 \mathrm{~min}$. The platelets were washed with PBS 3 times, followed by flow cytometry. The platelets were identified according to their forward scatter and CD41a antigen. The percentage of CD62p-positive platelets, an indicator of platelet activation, was analyzed.

Detection of Platelet Aggregation Using a PL-12 Platelet

Function Analyzer

Centrifugation was performed on the apheresis platelet suspensions at 4,000 rpm for $15 \mathrm{~min}$, and the supernatant was discarded. The platelet precipitate was washed with PBS 3 times, 5 min each time. RAPA (200 nM/L) and IGF-1 (50 nM/L) were added for pretreatment, respectively. The pretreated samples were loaded into a rotary mixer and mixed well. Then, $400 \mu \mathrm{L}$ of the sample was collected into Eppendorf tubes containing the inducers adenosine diphosphate (ADP; Sinnowa, China) and arachidonic acid (AA; Sinnowa, China), respectively. The two portions of the sample were loaded to channel 1 and 2 of the testing instruments, respectively, and the "start" button was clicked. After the testing was over, the maximum platelet aggregation rate after the induction by ADP and AA was measured.

\section{Detection of Expression Levels of Platelet Cytokines and}

Growth Factors

The expression levels of cytokines and growth factors were measured by ELISA. The apheresis platelet suspensions were centrifuged at 4,000 rpm for $15 \mathrm{~min}$, and the supernatant was discarded. The platelet precipitate was washed with PBS 3 times, 5 min each time. Then, it was pretreated with RAPA (200 nM/L) and IGF-1 (50 nM/L), respectively. Then, $400 \mu \mathrm{L}$ of the sample was collected into Eppendorf tubes containing inducers ADP and AA, respectively. The induced platelets were tested for expression levels of vascular endothelial growth factor (VEGF), platelet-derived growth factor (PDGF), transforming growth factor beta 1 (TGF- $\left.\beta_{1}\right)$, tumor necrosis factor alpha (TNF- $\alpha$ ), interleukin-6 (IL-6), and IL-8 according to the requirements of the corresponding ELISA kit (Boster, China).

Evaluation of the Efficacy of Clinical Platelet Transfusion

The barcodes of apheresis platelets detected for autophagic activity were collected. The patients (Table 2) receiving the transfusion of these apheresis platelets were tracked using the transfusion information system. Platelet counts before transfusion and at $24 \mathrm{~h}$
Table 2. General clinical data of patients $(n=84)$ with platelet transfusion

\begin{tabular}{lll}
\hline & Cases, $n(\%)$ & $p$ value \\
\hline Age & & 0.544 \\
$\quad \geq 45$ years & $45(53.6)$ & \\
$\quad<45$ years & $39(46.4)$ & 0.529 \\
Gender & & \\
$\quad$ Male & $44(52.4)$ & \\
$\quad$ Female & $40(47.6)$ & 0.621 \\
Weight & & \\
$\quad \geq 50$ kg & $50(59.5)$ & \\
$\quad<50$ kg & $38(40.5)$ & \\
Clinical diagnosis* & & \\
$\quad$ AL & $30(35.7)$ & \\
$\quad$ MDS & $16(19.0)$ & \\
$\quad$ AA & $13(15.5)$ & \\
$\quad$ MM & $25(29.8)$ & \\
Fever & $0(0)$ & \\
Hypersplenism & $0(0)$ & \\
Platelet antibody (negative) & $84(100)$ & \\
1st transfusion (platelet) & $84(100)$ & \\
ABO compatible & $84(100)$ & \\
\hline
\end{tabular}

$\mathrm{AL}$, acute leukemia; MDS, bone marrow hyperplasia; AA, aplastic anemia; MM, multiple myeloma. ${ }^{*}$ All patients were diagnosed with hematologic disease for the first time and were treated for the first time.

after transfusion were determined for the patients using a LIS information system. Efficacy was evaluated in terms of corrected count increment (CCI) after platelet transfusion.

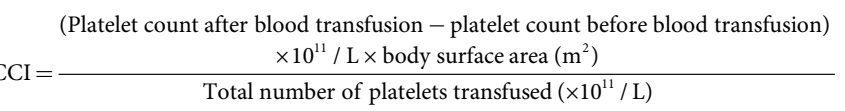

Body surface area $=0.0061 \times$ height $($ in $\mathrm{cm})+0.128 \times$ weight $($ in $\mathrm{kg})-0.01529$. Platelet transfusion was considered ineffective with 1 -h CCI $<7.5$ or 24 -h CCI $<4.5$ after transfusion.

\section{Statistical Analyses}

Statistical analyses were performed using SPSS 19.0 software. Data from 3 independent experiments were represented as mean \pm standard deviation. The means of the two groups were compared using a $t$ test. The means of multiple groups were compared using ANOVA. A $p$ value $<0.05$ indicated significant difference.

\section{Results}

\section{Baseline Autophagy Was Weak in Platelets}

To analyze whether autophagy could influence the functions of apheresis platelets after different storage durations, we first studied whether there was baseline autophagy in platelets. Western blot indicated weak expressions of Beclin 1 and LC3-II proteins in fresh platelets, which are markers of weak autophagy in platelets. However, the autophagic activity was much lower than that in the karyotes (Fig. $1 ; p<0.05$ ). 


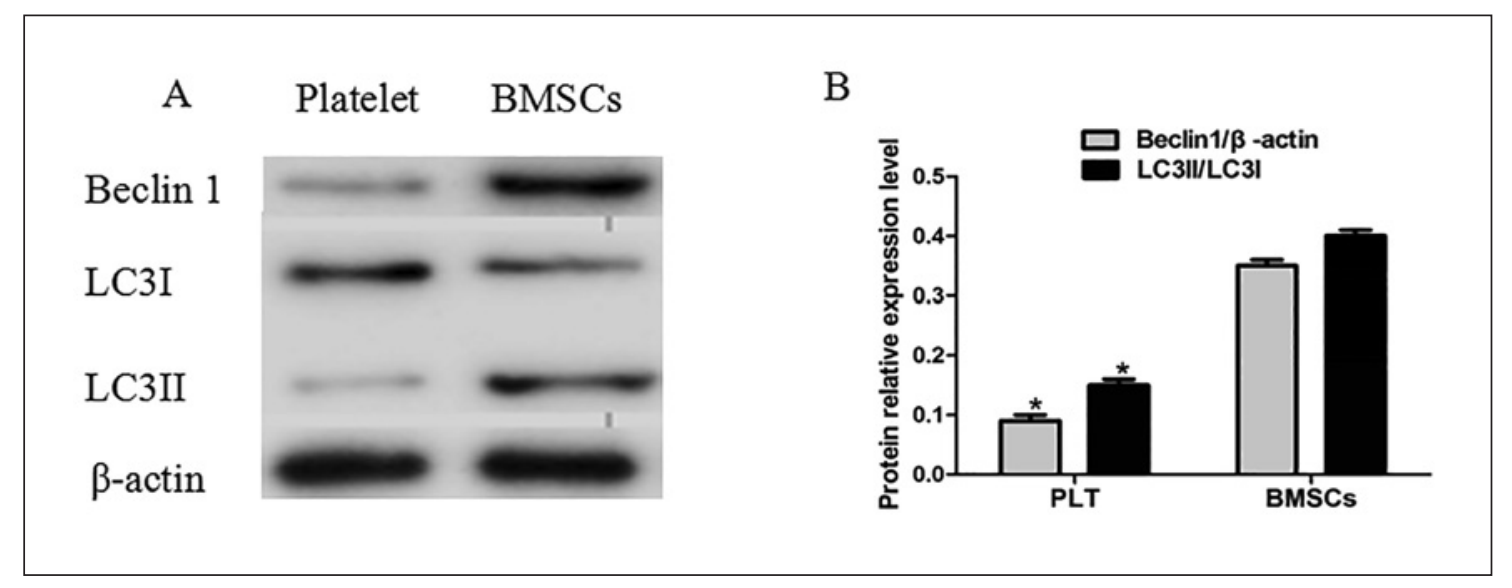

Fig. 1. Expressions of Beclin 1 and LC3 proteins in platelets detected by Western blot. ${ }^{*}$ Indicates significant difference as compared with bone mesenchymal stem cells $(p<0.05)$.

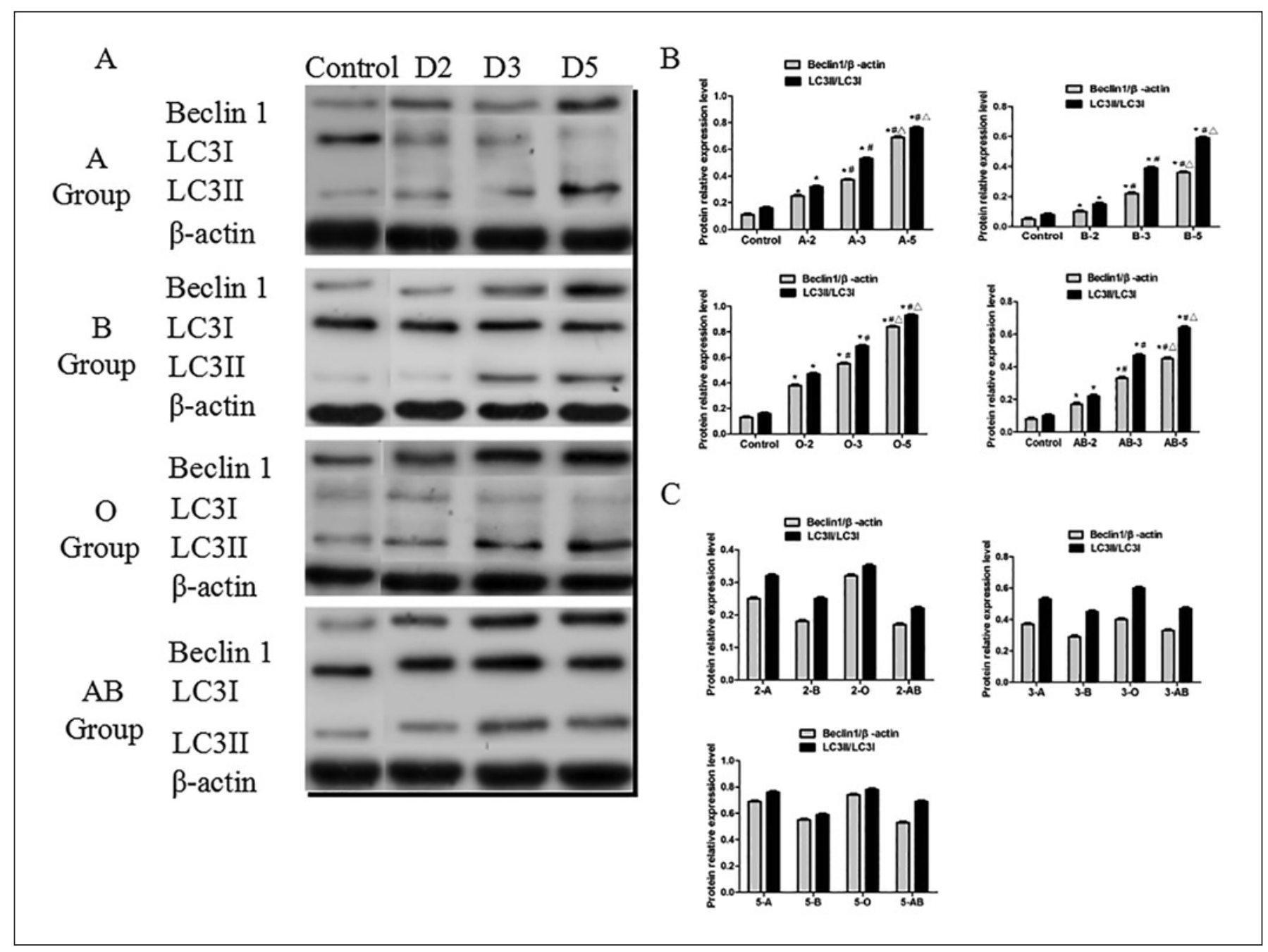

Fig. 2. Expressions of Beclin 1 and LC3 proteins in apheresis platelets detected by Western blot. B, C Expressions of autophagy-related proteins after different storage durations and for group A, B, O, and AB blood; * indicates comparison with the control group (fresh platelets); \# indicates comparison with the apheresis platelets after storage for 2 days; $\triangle$ indicates comparison with the apheresis platelets after storage for 3 days $(p<0.05)$. 


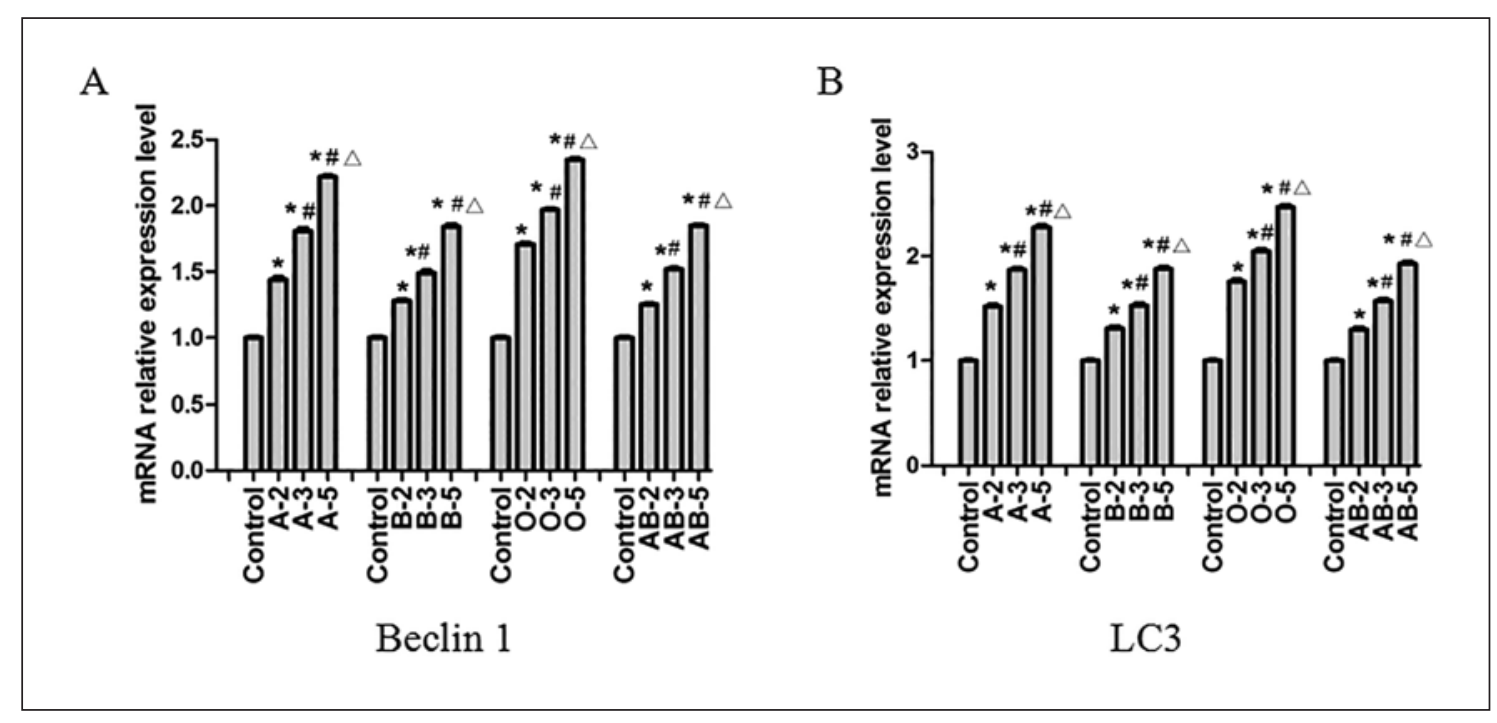

Fig. 3. Expressions of Beclin 1 (A) and LC3 (B) genes after different storage durations defected by RT-qPCR; * indicates comparison with the control group (fresh platelets); ${ }^{*}$ indicates comparison with the apheresis platelets after storage for 2 days; ${ }^{\triangle}$ indicates significant difference as compared with the apheresis platelets after storage for 3 days $(p<0.05)$.

\section{Storage Duration Influenced Autophagic Activity of Apheresis Platelets}

It has already been demonstrated that there is baseline autophagy in platelets. To determine whether storage duration influenced autophagic activity of platelets in vitro, we detected the expressions of autophagy-related proteins and genes in apheresis platelets after storage for 2, 3, and 5 days, respectively. According to the results of Western blot, Beclin 1 and LC3 proteins were presented in all apheresis platelet samples, and their expressions were all significantly higher than those in the control group (fresh platelets). Autophagic activity of platelets is time dependent. That is, the expressions of Beclin 1 and LC3-II proteins increased significantly with the prolongation of storage duration (for type A, B, O, and AB blood) (Fig. 2A, $\mathrm{B} ; p<0.05)$. According to RT-qPCR, there was also a significant upregulation in Beclin 1 and LC3 genes in apheresis platelets with the prolongation of storage duration (for type A, B, O, and AB blood). The expression intensity was the highest in 5-day-old platelets, followed by that in 3- and 2-day-olds, and the control group had the weakest expression (Fig. 3; $p<0.05$ ).

\section{Influence of $A B O$ Blood Group on Autophagic Activity of Apheresis Platelets}

Western blot revealed that the expressions of Beclin 1 and LC3-II in platelets (whether after 2, 3, or 5 days) for type $\mathrm{A}$ and $\mathrm{O}$ blood were upregulated as compared with those for type $\mathrm{B}$ and $\mathrm{AB}$ blood, but not in a significant way (Fig. 2A, C). RT-qPCR results indicated that the expressions of Beclin 1 and LC3 genes in apheresis platelets of type $\mathrm{A}$ and $\mathrm{O}$ blood were also insignificantly higher as compared with those of type $\mathrm{B}$ and $\mathrm{AB}$ blood (Fig. 4).

\section{Influence of RAPA and IGF-1 on the PI3K/AKT/}

mTOR and JAK/STAT Signaling Pathway

To identify the signaling pathway that influences autophagy in platelets, we detected the changes in the expressions of proteins related to the $\mathrm{PI} 3 \mathrm{~K} / \mathrm{AKT} / \mathrm{mTOR}$ and JAK/STAT signaling pathway after pretreatment with RAPA or IGF- 1 for 5 days, respectively. Western blot indicated that after RAPA pretreatment, there was a significant downregulation in p-mTOR, $\mathrm{p}$-AKT, and $\mathrm{p}$ $\mathrm{PI} 3 \mathrm{~K}$ proteins in apheresis platelets of type $\mathrm{O}$ blood (Fig. 5A; $p<0.05$ ). After IGF-1 pretreatment, there was a significant upregulation in $\mathrm{p}-\mathrm{mTOR}$, $\mathrm{p}-\mathrm{AKT}$, and $\mathrm{p}-\mathrm{PI} 3 \mathrm{~K}$ proteins in apheresis platelets of type O blood (Fig. 5A; $p<0.05)$. Western blot indicated that after RAPA and IGF-1 pretreatment, there was no significant change in p-JAK1 and p-STAT1 proteins in apheresis platelets of type O blood (Fig. 5C). These results implied that RAPA and IGF-1 pretreatments could regulate the PI3K/AKT/ mTOR signaling pathway.

\section{Influence of RAPA and IGF-1 on the Autophagic \\ Activity of Apheresis Platelets}

To study whether RAPA and IGF-1 influenced the autophagic activity of apheresis platelets, RAPA and IGF-1, respectively, were co-cultured with apheresis platelets of type $\mathrm{O}$ blood for $2 \mathrm{~h}$ after different storage durations. Changes in autophagic activity in apheresis platelets of type O blood were detected before and after pretreatment. 
A

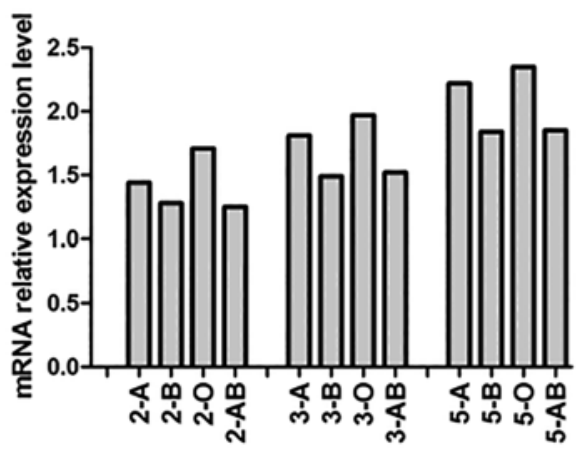

Beclin 1
B

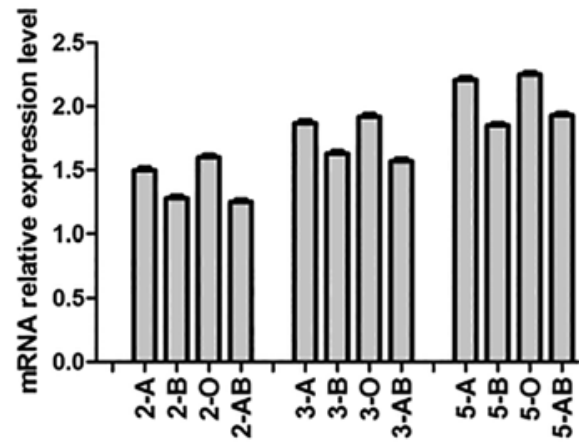

LC3

Fig. 4. Expressions of Beclin 1 (A) and LC3 (B) genes in apheresis platelets based on ABO blood group as detected by RT-qPCR.

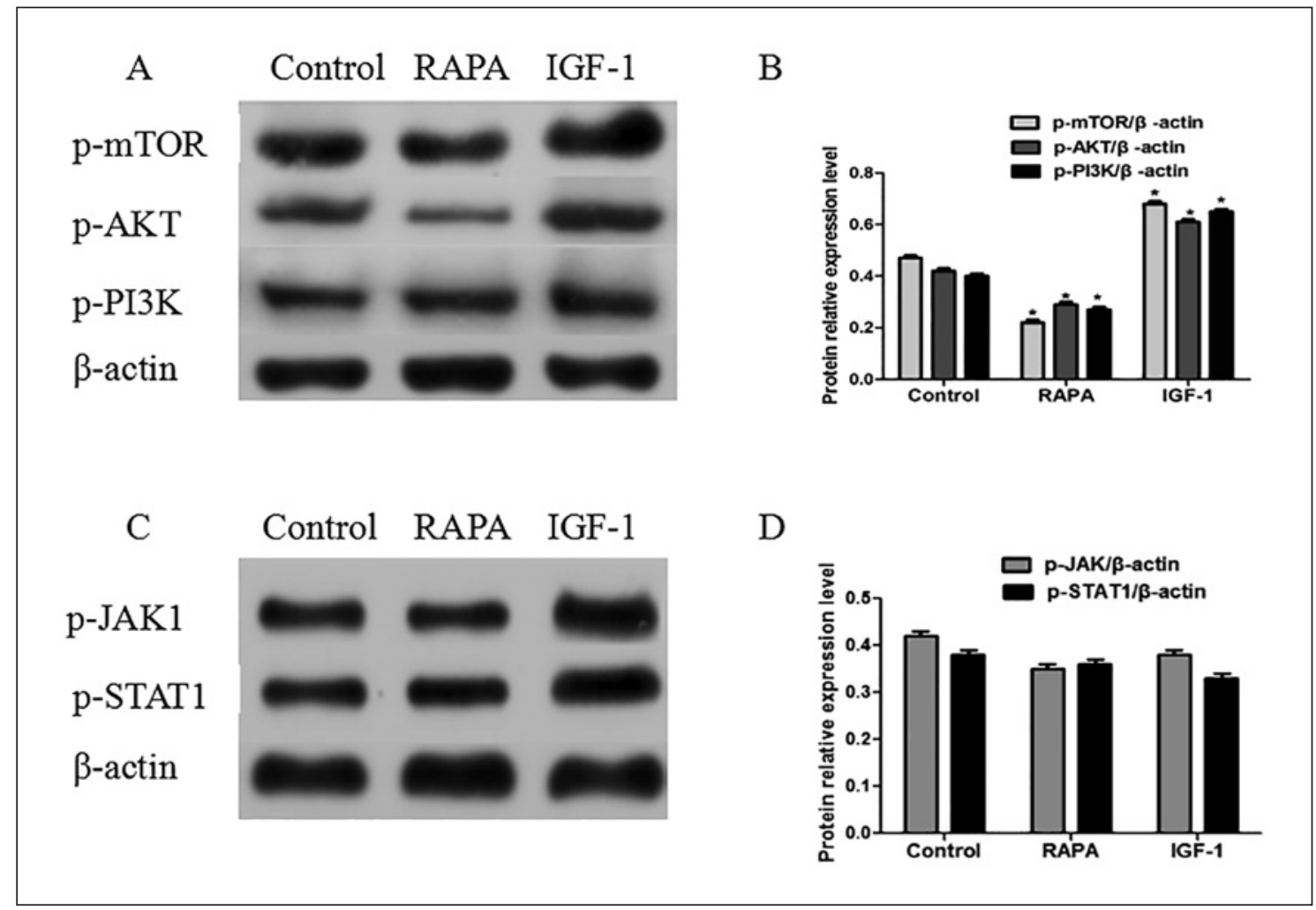

Fig. 5. Expressions of proteins related to the PI3K/AKT/mTOR and JAK/STAT signaling pathway after pretreatments with RAPA and IGF-1, respectively, as detected by Western blot; * indicates significant difference as compared with the control group (non-pretreated apheresis platelets) $(p<0.05)$.

According to Western blot, after pretreatment with RAPA, the mTOR inhibitor, there was a significant upregulation of Beclin 1 and LC3-II proteins in apheresis platelets, indicating that RAPA stimulated the autophagy in platelets (Fig. $6 ; p<0.05$ ). In contrast, after pretreatment wit IGF-1, the mTOR agonist, Beclin 1 and LC3-II proteins in the apheresis platelets were downregulated significantly, which indicated the inhibition of autophagy 


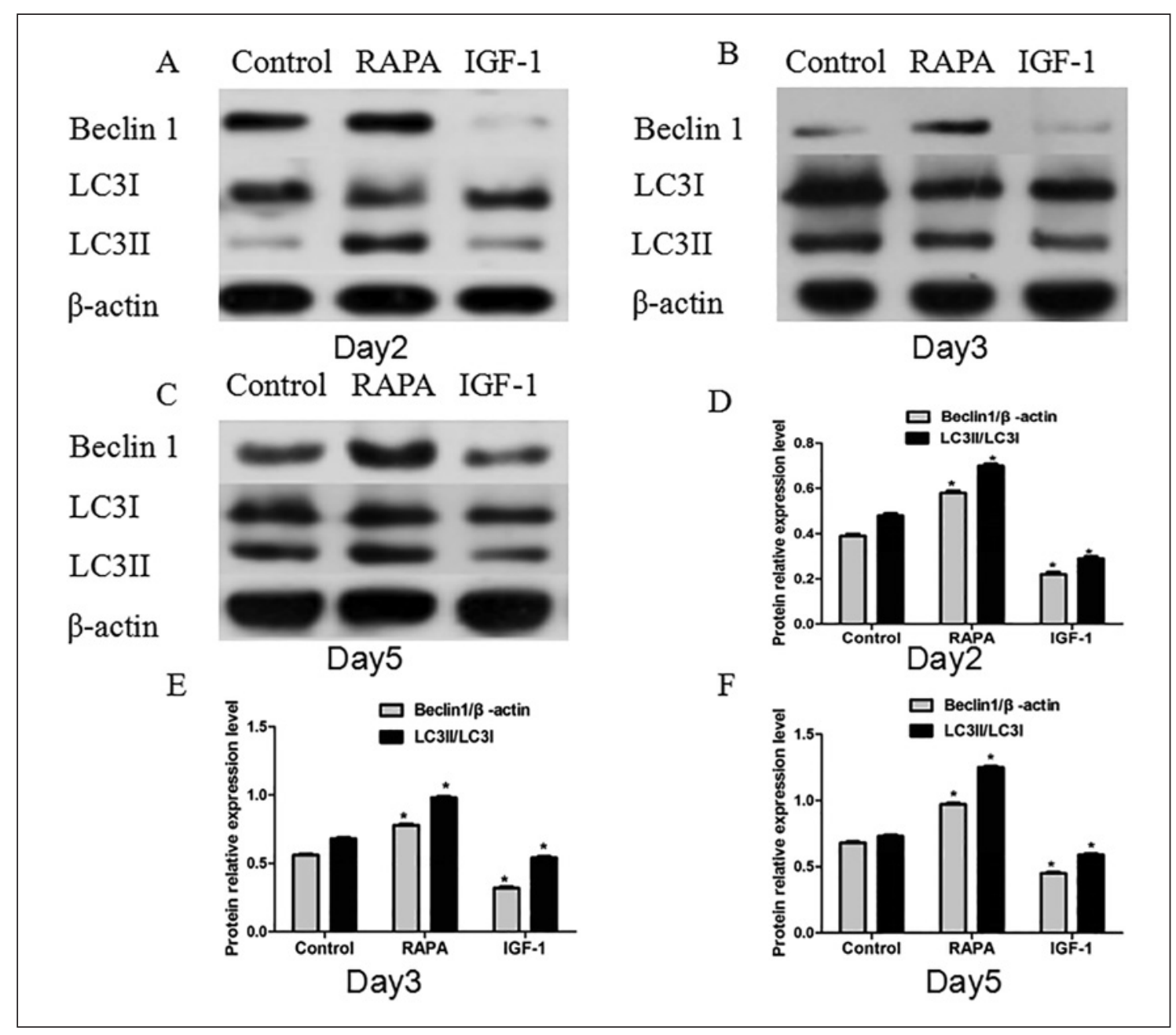

Fig. 6. Beclin 1 and LC3 protein expressions in apheresis platelets after pretreatment with RAPA and IGF-1 as detected by Western blot; $\mathbf{A}$ and $\mathbf{D}, \mathbf{B}$ and $\mathbf{E}$, and $\mathbf{C}$ and $\mathbf{F}$ are the expressions of autophagy-related proteins in apheresis platelets of type $\mathrm{O}$ blood after 2,3 , and 5 days of storage, respectively; ${ }^{*}$ indicates significant difference as compared with the control group (non-pretreated apheresis platelets) $(p<0.05)$.

in platelets by IGF-1 (Fig. $6 ; p<0.05$ ). The results above demonstrated the negative regulatory role of the mTOR signaling pathway in platelet autophagy. Immunofluorescence detection of Beclin 1 and LC3 found that the autophagic activity of platelets was regulated by RAPA and IGF-1 (Fig. 7). According to RT-qPCR, after pretreatment with RAPA and IGF-1, the Beclin 1 and LC3-II genes in platelets were upregulated and downregulated, respectively (Fig. 8; $p<0.05$ ).

\section{Influence of Autophagy on Platelet Activation and Aggregation}

The results of flow cytometry and PL-12 platelet function analyzer indicated that with the prolongation of storage time, the activation degree increased significantly in the apheresis platelets of type O blood, while the platelet aggregation rate decreased significantly. After RAPA pre- treatment, the percentage of CD62p-positive platelets was significantly higher for type O blood compared to the control group, indicating an increase in platelet activation after RAPA pretreatment. After IGF-1 pretreatment, the percentage of CD62p-positive platelets was significantly lower for type O blood compared to the control group, indicating a reduction in the activation level following IGF-1 pretreatment (Fig. 9; $p<0.05$ ). After RAPA pretreatment, the maximum platelet aggregation rates induced by ADP and AA were much lower for type $\mathrm{O}$ blood compared to the control group. However, after IGF-1 pretreatment, the maximum platelet aggregation rates induced by ADP and AA were much higher for type $\mathrm{O}$ blood compared to the control group (Fig. 10; $p<0.05$ ). The above results indicated that the activation and aggregation of apheresis platelets were regulated by autophagic activity. 


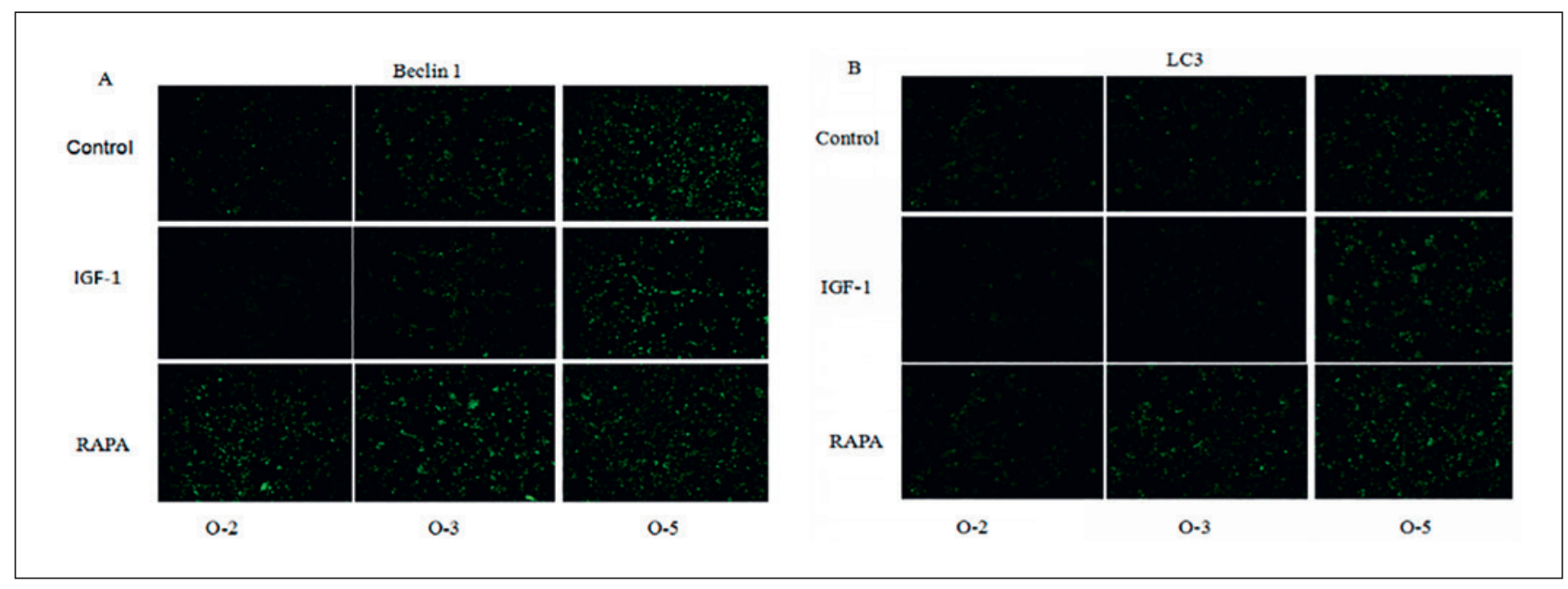

Fig. 7. Immunofluorescence detection of Beclin 1 and LC3 proteins in apheresis platelets after pretreatment with RAPA and IGF-1, respectively. Scale bar $=50 \mu \mathrm{m}$.

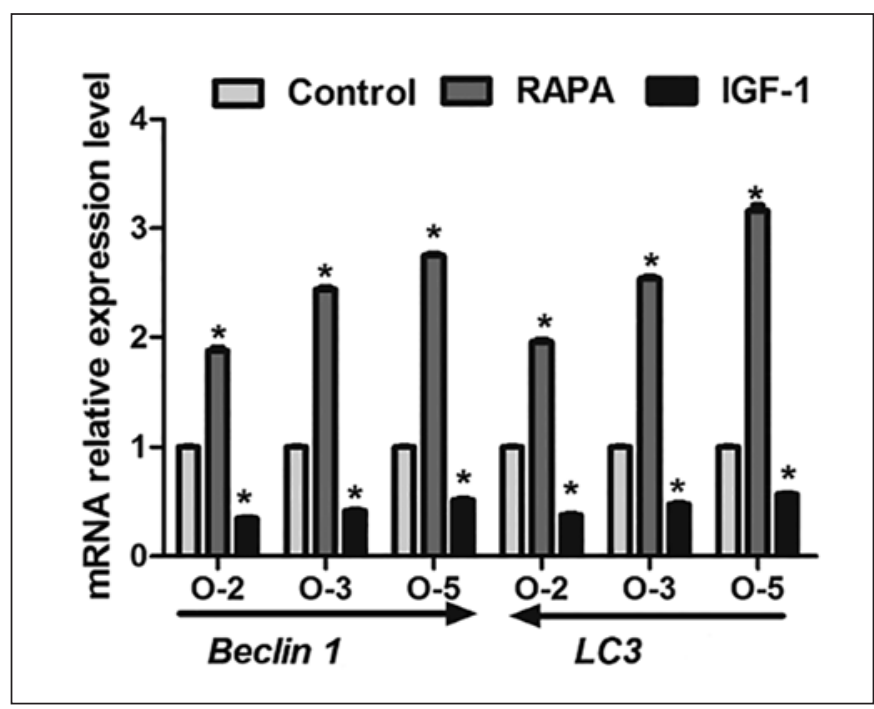

Fig. 8. Expressions of Beclin 1 and LC3 genes in apheresis platelets after pretreatment with RAPA and IGF-1, respectively, as detected by RT-qPCR; * indicates significant difference as compared with the control group (non-pretreated apheresis platelets) $(p<0.05)$.

\section{Influence of RAPA and IGF-1 on Expression of \\ Platelet Cytokines and Growth Factors}

When platelets are activated, various growth factors and cytokines are released. This study examined changes in VEGF, PDGF, TGF- $\beta_{1}$, TNF- $\alpha$, IL- 6 , and IL- 8 during platelet activation. After RAPA pretreatment, the expression levels of VEGF, PDGF, TGF- $\beta_{1}$, TNF- $\alpha$, IL- 6 , and IL- 8 induced by ADP and AA were much higher for type O blood compared to the control group. However, after IGF-1 pretreatment, the expression levels of VEGF, PDGF, TGF- $\beta_{1}$, TNF- $\alpha$, IL- 6 , and IL- 8 induced by ADP and AA were much lower for type O blood compared to the control group (Fig. 11; $p<0.05$ ).

Autophagic Activity Influenced the Efficacy of Clinical Platelet Transfusion by Changing Platelet Function

Activation and aggregation of apheresis platelets are regulated by autophagic activity. Therefore, changes in autophagic activity would also influence the efficacy of clinical platelet transfusion. CCI was calculated based on the platelet count before transfusion (apheresis platelets detected for autophagic activity) and at $24 \mathrm{~h}$ after transfusion. Nine cases were excluded as the platelet count was not available at $24 \mathrm{~h}$ after blond transfusion. Thus, a correlation analysis was performed between the LC3-II/LC3I ratio and CCI after blood transfusion for the remaining 84 patients. The results showed that the LC3-II/LC3-I ratio correlated negatively with the CCI (Fig. $12 ; r^{2}=0.8057$, $p<0.0001$ ). That is, the higher the LC3-II/LC3-I ratio (the higher the autophagic activity), the lower the CCI at $24 \mathrm{~h}$ after transfusion (the worse the efficacy of clinical platelet transfusion). These results were indirect proof that changes in autophagic activity of apheresis platelets might influence the efficacy of clinical platelet transfusion.

\section{Discussion}

Autophagy is an inherent protective mechanism in organisms, and weak baseline autophagy can be found in nearly all eukaryocytes. Autophagy has been rarely studied in akaryotes. Until 1970s, greater academic attention was given to platelet autophagy. Autophagy is not only present in resting platelets, but it also affects platelet activation and aggregation [13-15]. However, the existing 
A

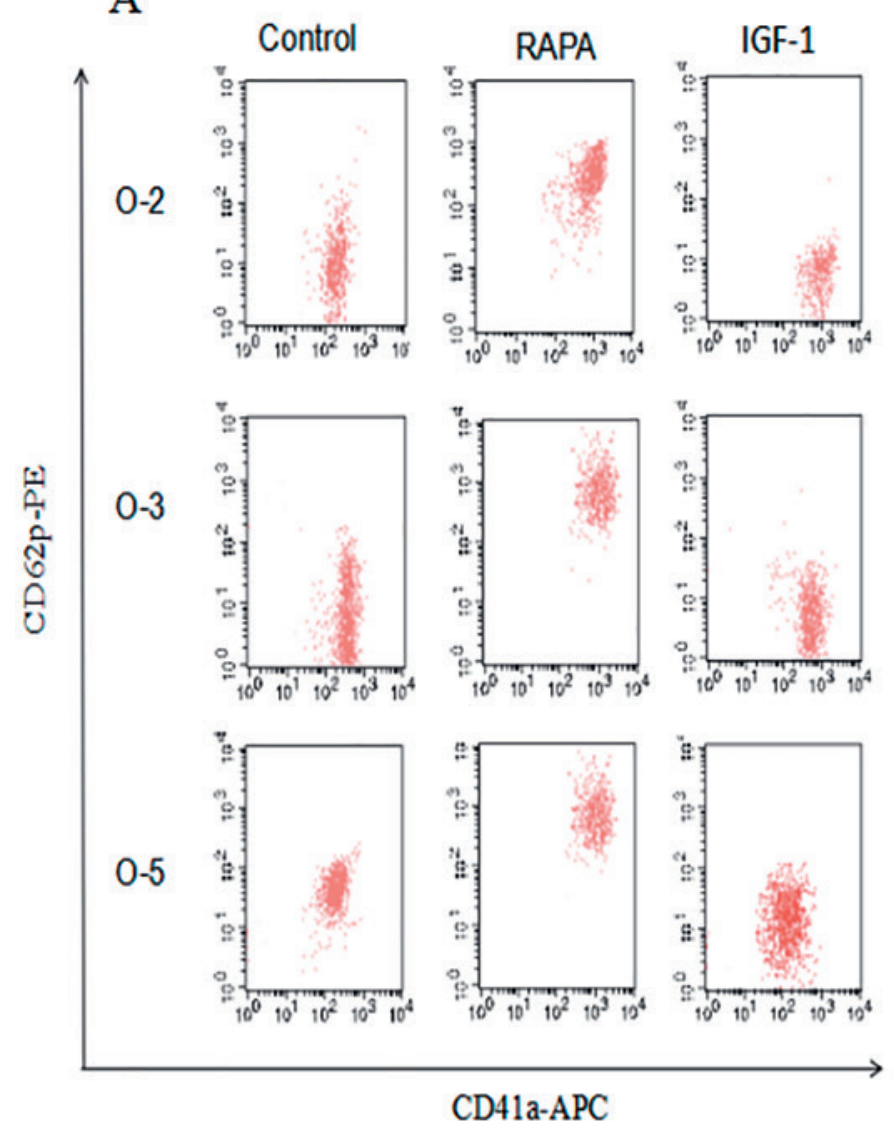

B

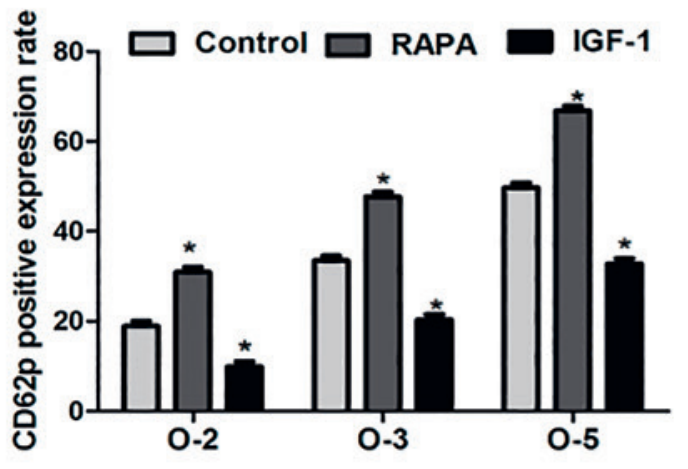

C

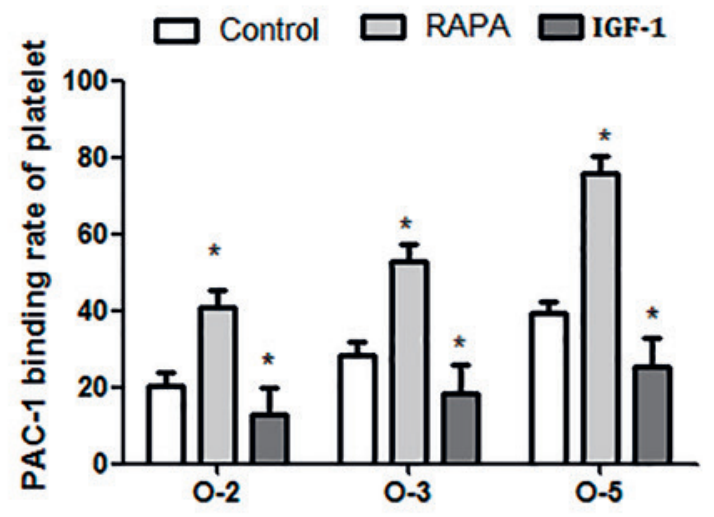

Fig. 9. Activation levels of apheresis platelets pretreated by RAPA and IGF-1 as detected by flow cytometry; * indicates significant difference as compared with the control group (non-pretreated apheresis platelets) $(p<0.05)$.

A

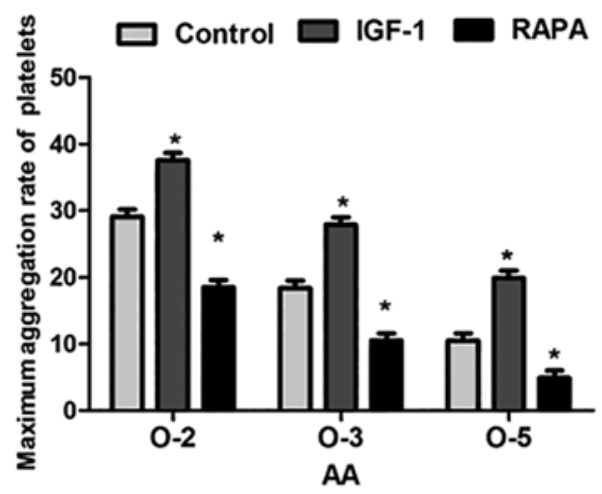

B

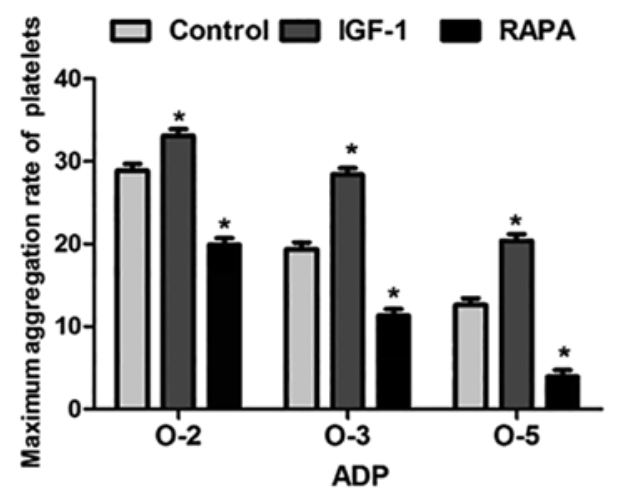

Fig. 10. The maximum platelet aggregation rate after pretreatment with RAPA and IGF-1 as detected by the PL12 platelet function analyzer; ${ }^{*}$ indicates significant difference as compared with the control group (non-pretreated apheresis platelets) $(p<0.05)$. The reference ranges for the maximum platelet aggregation rate after induction with AA and ADP were $40-80$ and $35-75$, respectively. 
A

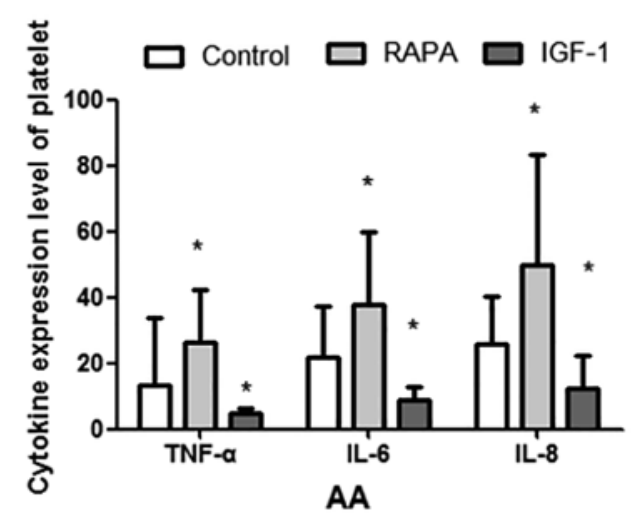

B

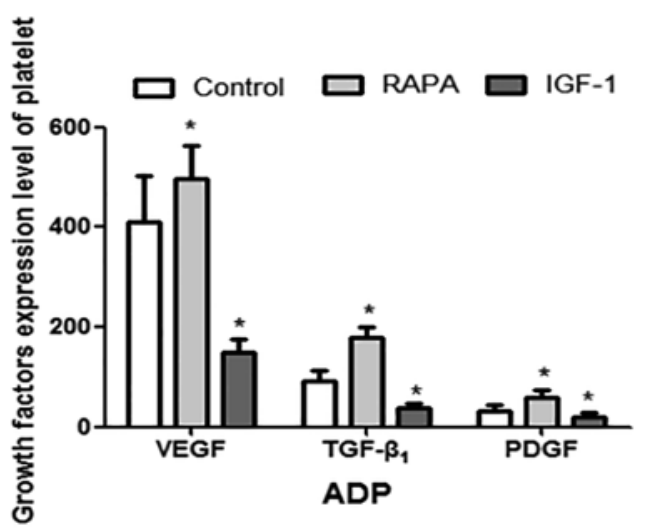

Fig. 11. The expression levels of platelet cytokine and growth factors after pretreatment with RAPA and IGF-1; * indicates significant difference as compared with the control group (non-pretreated apheresis platelets) $(p<$ $0.05)$.

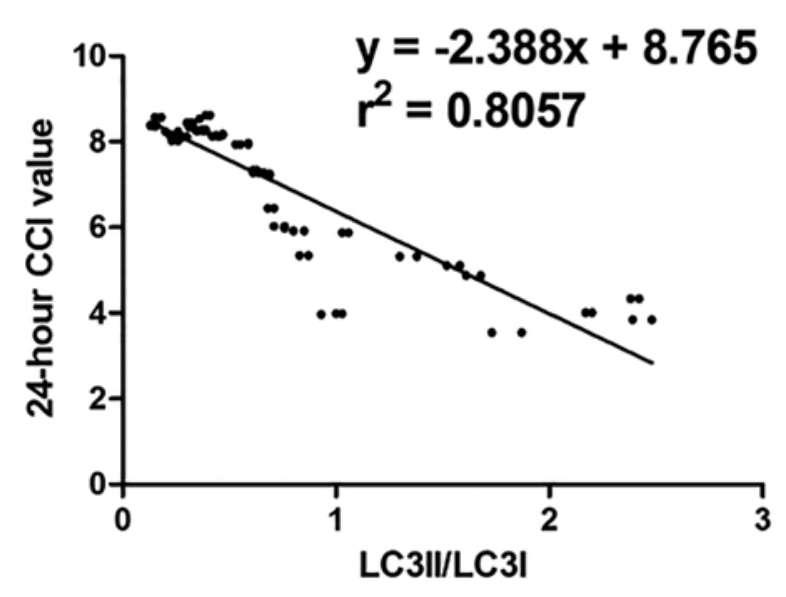

Fig. 12. Correlation analysis between autophagic activity of apheresis platelets and efficacy of clinical platelet transfusion.

experiments are generally performed in fresh platelets. It remains unknown whether autophagic activity of apheresis platelets of different blood types affects platelet functions and efficacy of platelet transfusion after storage for different durations. Few studies have been conducted looking at these aspects so far.

Beclin 1 and LC3 are considered as the basis and markers of autophagy, and their expressions are significantly upregulated under autophagy. By detecting fresh platelets for autophagic activity, it was found that the autophagyrelated proteins Beclin 1 and LC3 were obviously expressed, which was in agreement with the existing findings with karyocytes. However, the autophagic activity in fresh platelets was much lower than that in the karyocytes.
The presence of autophagy-related proteins in fresh platelets was the basis for autophagy. Beclin 1 and LC3 proteins and genes were also detected in apheresis platelets. Since genetic manipulation is impossible with platelets, changes in autophagic activities of platelets could be only studied by using agonists or inhibitors. Therefore, RAPA and IGF-1 were, respectively, applied to the apheresis platelets. As a result, the autophagic activity increased or decreased. As demonstrated by the above results, platelets (akaryocytes) can also undergo autophagy like karyocytes.

Except for erythrocyte membrane, the membranes of many types of cells express $\mathrm{ABO}$ blood group antigens at a high frequency, including epithelial cells, sensory neurons, vascular endothelial cells, and platelets [16]. The $\mathrm{ABO}$ blood group, as the most important blood type system, has been found in significant correlations with autoimmune diseases, cardiovascular diseases, infectious diseases, and tumors [17-20]. The ABO blood group antigen is also expressed on the platelet membrane, from which we speculate that the autophagic activity of apheresis platelets may be influenced by ABO blood types. By detecting the autophagic activity of apheresis platelets for different blood types, it was found that the expressions of autophagy-related proteins for type $\mathrm{A}$ and $\mathrm{O}$ blood were only insignificantly higher as compared with type $\mathrm{B}$ and $\mathrm{AB}$ blood. The $\mathrm{ABO}$ blood group affected the autophagic activity of apheresis platelets only to a minor extent, and a larger sample size was needed to verify this.

The mechanism of autophagy is very complex and regulated positively or negatively by different signaling pathways [21, 22]. The mTOR (mTOR1 and mTOR2) signaling pathway is most thoroughly understood in relation to autophagy, especially mTOR1. It has been reported that active mTOR kinase is also present in platelets [23]. 
RAPA is a specific inhibitor of mTOR that can induce autophagy and, therefore, is frequently used in the researches on autophagy [24]. IGF-1 is an agonist of mTOR, which can regulate autophagy [25]. In the present study, RAPA and IGF- 1 were used to pretreat apheresis platelets after storage for 2, 3, or5 days, respectively, and the autophagic activity was determined after the pretreatment. As compared with the non-pretreated platelets (control group), the expressions of p-mTOR, p-AKT, and p-PI3K were downregulated significantly after RAPA pretreatment, and the autophagic activity increased significantly. On the contrary, after IGF-1 pretreatment, the expressions of $\mathrm{p}$-mTOR, $\mathrm{p}$-AKT, and $\mathrm{p}-\mathrm{PI} 3 \mathrm{~K}$ were upregulated significantly, while the autophagic activity decreased considerably. The above results demonstrate the regulatory role of the PI3K/AKT/mTOR signaling pathway in the autophagic activity of apheresis platelets.

Activation of apheresis platelets during storage seems to be inevitable, though its mechanism is not fully known. Autophagy as a widely present protective mechanism is involved in many physiological activities of cells, organelles, and tissues [26]. Our preliminary experiments showed that the apheresis platelets in storage underwent autophagy, which was regulated by the $\mathrm{PI} 3 \mathrm{~K} / \mathrm{AKT} / \mathrm{mTOR}$ signaling pathway. From this it was inferred that autophagy might also participate in platelet activation and aggregation. To test this hypothesis, flow cytometry and PL12 platelet function analyzer were used to assess the activation and aggregation functions of apheresis platelets before and after RAPA and IGF-1 pretreatment, respectively. The results showed that the activation level of apheresis platelets increased, while platelet aggregation decreased after RAPA pretreatment. In contrast, after IGF-1 pretreatment, the platelet activation level decreased, while the aggregation level increased. The reason for this phenomenon may be that after the blockage of autophagic degradation with the use of specific inhibitor IGF-1, there was a constant accumulation of abnormal or injured proteins in apheresis platelets after long-term storage. As the dynamic balance is disrupted, the signals related to platelet activation were blunted. Our experimental results indicate that autophagy regulated the activation of apheresis platelets and served as an important mechanism of the activation of apheresis platelets. Our results coincided with those by Aslan et al. [27], who found that the mTOR signaling pathway could regulate platelet activation and aggregation. However, our results seemed to contradict the studies by Ouseph et al. [28] and Feng et al. [12]. According to Ouseph et al. [28], the activation of fresh platelets resulted in a reduction of LC3-II. Feng et al. [12] found that the platelet aggregation function declined after inhibiting autophagy, which might be due to the apheresis procedure and long-term storage of platelets in vitro.
An important influence factor of efficacy of platelet transfusion is platelet activation during storage. Our experimental results demonstrate that platelet activation releases a variety of cytokines and growth factors, which may be the reason why activated platelets affect the efficacy of platelet transfusion. The existing experiments have shown that autophagy affects the activation and aggregation functions of apheresis platelets. From this, it can be inferred that autophagy also influences the efficacy of transfusion using apheresis platelets. However, apheresis platelets with autophagic intervention cannot be transfused into patients for ethical reasons. Therefore, the relationship between autophagy and efficacy of platelet transfusion was discussed based on the LC3-II/LC3-I ratio and CCI in apheresis platelets. The results showed that the higher the autophagic activity, the worse the efficacy of platelet transfusion. This is indirect evidence that autophagy affects the efficacy of platelet transfusion. Only in vitro experiments were conducted in this study, and the future research direction will be the influence of autophagy on platelet functions and efficacy of platelet transfusion in animal experiments.

This study has certain limitations. We have only selected the classical signaling pathway in the process of detecting autophagy and did not cover all signaling pathways of autophagy. In addition, we only performed in vitro cell experiments. This study will be followed by animal experiments, knocking out relevant target genes to further verify the accuracy of the results.

To conclude, long-term storage of apheresis platelets can significantly enhance autophagic activity. Pretreatment with RAPA and IGF-1 can further regulate the autophagic activity of apheresis platelets. Changes in autophagic activity will affect the efficacy of platelet transfusion by influencing the activation and aggregation functions of apheresis platelets. Our findings provide theoretical basis for animal experiments and also for avoiding blood waste.

\section{Statement of Ethics}

All experiments and information collection were approved by the hospital's Ethics Committee (approval number: 2017122).

\section{Disclosure Statement}

The authors report no conflicts of interest.

\section{Author Contributions}

Hao Tang carried out the experimental work. Meng Gao carried out the data collection and interpretation. Hao Tang, Yunfeng $\mathrm{Fu}$, and Rong Gui carried out study design. Xianjun Ma participated in the study design. 


\section{References}

1 Benson K, Fields K, Hiemenz J, Zorsky P, Ballester O, Perkins J, et al. The platelet-refractory bone marrow transplant patient: prophylaxis and treatment of bleeding. Semin Oncol. 1993 Oct;20(5 Suppl 6):102-9.

2 Quaglietta A, Di Saverio M, Lucisano G, Accorsi P, Nicolucci A. Development of the Platelet Efficacy Score (PEscore) to predict the efficacy of platelet transfusion in oncohematologic patients. Transfusion. 2017 Apr; 57(4):905-12.

3 Shapira S, Friedman Z, Shapiro H, Presseizen $\mathrm{K}$, Radnay J, Ellis MH. The effect of storage on the expression of platelet membrane phosphatidylserine and the subsequent impacton the coagulant function of stored platelets. Transfusion. 2000 Oct;40(10):1257-63.

4 Mittal K, Kaur R. Platelet storage lesion: an update. Asian J Transfus Sci. 2015 Jan-Jun; 9(1):1-3.

5 Heras-Sandoval D, Pérez-Rojas JM, Hernández-Damián J, Pedraza-Chaverri J. The role of $\mathrm{PI} 3 \mathrm{~K} / \mathrm{AKT} / \mathrm{mTOR}$ pathway in the modulation of autophagy and the clearance of protein aggregates in neurodegeneration. Cell Signal. 2014 Dec;26(12):2694-701.

6 Kuballa P, Nolte WM, Castoreno AB, Xavier RJ. Autophagy and the immune system. Annu Rev Immunol. 2012;30(1):611-46.

7 Deretic V, Saitoh T, Akira S. Autophagy in infection, inflammation and immunity. Nat Rev Immunol. 2013 Oct;13(10):722-37.

8 Heath RJ, Xavier RJ. Autophagy, immunity and human disease. Curr Opin Gastroenterol. 2009 Nov;25(6):512-20.

9 Nencioni A, Cea M, Montecucco F, Longo VD, Patrone F, Carella AM, et al. Autophagy in blood cancers: biological role and therapeutic implications. Haematologica. 2013 Sep;98(9):1335-43.
10 Zhang J, Wu K, Xiao X, Liao J, Hu Q, Chen H, et al. Autophagy as a regulatory component of erythropoiesis. Int J Mol Sci. 2015 Feb;16(2): 4083-94.

11 Lewis JC, Maldonado JE, Mann KG, Moertel CG. Ultrastructural cytochemistry of platelets and megakaryocytes in the carcinoid syndrome. Mayo Clin Proc. 1976 Sep;51(9):58593.

12 Feng W, Chang C, Luo D, Su H, Yu S, Hua W, et al. Dissection of autophagy in human platelets. Autophagy. 2014 Apr;10(4):642-51.

13 Cao Y, Cai J, Zhang S, Yuan N, Li X, Fang Y, et al. Loss of autophagy leads to failure in megakaryopoiesis, megakaryocyte differentiation, and thrombopoiesis in mice. Exp Hematol. 2015 Jun;43(6):488-94.

14 Liu Y, Hu M, Luo D, Yue M, Wang S, Chen X, et al. Class III PI3K Positively Regulates Platelet Activation and Thrombosis via PI(3)P-Directed Function of NADPH Oxidase. Arterioscler Thromb Vasc Biol. 2017 Nov;37(11): 2075-86.

15 Valet C, Severin S, Chicanne G, Laurent PA, Gaits-Iacovoni F, Gratacap MP, et al. The role of class I, II and III PI 3-kinases in platelet production and activation and their implication in thrombosis. Adv Biol Regul. 2016 May; 61:33-41.

16 Eastlund T. The histo-blood group ABO system and tissue transplantation. Transfusion. 1998 Oct;38(10):975-88.

17 Liumbruno GM, Franchini M. Beyond immunohaematology: the role of the ABO blood group in human diseases. Blood Transfus. 2013 Oct;11(4):491-9.

18 Franchini M, Mannucci PM. ABO blood group and thrombotic vascular disease. Thromb Haemost. 2014 Dec;112(6):1103-9.

19 Pelzer U, Klein F, Bahra M, Sinn M, Dörken $\mathrm{B}$, Neuhaus $\mathrm{P}$, et al. Blood group determinates incidence for pancreatic cancer in Germany. Front Physiol. 2013 May;4(4):118.
20 Ouyang PY, Su Z, Mao YP, Liu Q, Xie FY. Prognostic value of $\mathrm{ABO}$ blood group in southern Chinese patients with established nasopharyngeal carcinoma. Br J Cancer. 2013 Oct;109(9):2462-6.

21 Ravikumar B, Vacher C, Berger Z, Davies JE, Luo S, Oroz LG, et al. Inhibition of mTOR induces autophagy and reduces toxicity of polyglutamine expansions in fly and mouse models of Huntington disease. Nat Genet. 2004 Jun;36(6):585-95.

22 Tassa A, Roux MP, Attaix D, Bechet DM. Class III phosphoinositide 3-kinase-Beclin1 complex mediates the amino acid-dependent regulation of autophagy in $\mathrm{C} 2 \mathrm{C} 12$ myotubes. Biochem J. 2003 Dec;376(Pt 3):577-86.

23 Weyrich AS, Denis MM, Schwertz H, Tolley ND, Foulks J, Spencer E, et al. mTOR-dependent synthesis of Bcl-3 controls the retraction of fibrin clots by activated human platelets. Blood. 2007 Mar;109(5):1975-83.

24 Kim YC, Guan KL. mTOR: a pharmacologic target for autophagy regulation. J Clin Invest. 2015 Jan;125(1):25-32.

25 Wang S, Gu K. Insulin-like growth factor 1 inhibits autophagy of human colorectal carcinoma drug-resistant cells via the protein kinase $\mathrm{B} /$ mammalian target of rapamycin signaling pathway. Mol Med Rep. 2018 Feb; 17(2):2952-6

26 Mizushima N, Komatsu M. Autophagy: renovation of cells and tissues. Cell. 2011 Nov; 147(4):728-41.

27 Aslan JE, Tormoen GW, Loren CP, Pang J, McCarty OJ. S6K1 and mTOR regulate Rac1driven platelet activation and aggregation. Blood. 2011 Sep;118(11):3129-36.

28 Ouseph MM, Huang Y, Banerjee M, Joshi S, MacDonald L, Zhong Y, et al. Autophagy is induced upon platelet activation and is essential for hemostasis and thrombosis. Blood. 2015 Sep;126(10):1224-33. 